\title{
Evaluación de las herramientas de autopercepción corporal de Sorensen versus Mash y Roche en la carrera de Diseño de Modas
}

Herrera-Hernández Pomeline Casdy*, Pérez-González Sara Eugenia*, Castorena-de la Torre Carla Estefanía*, Zapata-Cortés Ana Luisa*, Martín del Campo-Cervantes Judith**

\section{Resumen}

- La autopercepción es definida como la concepción que un - individuo tiene sobre sí mismo. Se ha observado que la cons- trucción de la imagen corporal está relacionada con factores - cognitivos, emocionales y conductuales. Cuando la imagen - corporal no concuerda con el cuerpo del sujeto existe una - distorsión de la imagen corporal, la cual se relaciona con des- órdenes alimenticios. Las escalas de evaluación para la auto- percepción corporal de Sorensen, y de Mash y Roche presen-

- tan al sujeto una serie de figuras femeninas y masculinas en

- progresión creciente en relación al índice de masa corporal

- (IMC). Objetivo: Evaluar las herramientas de autopercepción

- corporal de Sorensen versus Mash y Roche en estudiantes

- de la Licenciatura de Diseño de Modas en Indumentaria

- y Textiles de la Universidad Autónoma de Aguascalientes

- (UAA). Materiales y métodos: estudio analítico, transversal

- y observacional. Muestra no probabilística por cuota, obte-

- niendo 80 alumnos. Materiales: báscula de bioimpedancia

- Tanita BC-545, estadímetro mecánico SECA-2 17, formato

- de autopercepción corporal de Sorensen y de Mash y Roche.

- Resultados: El 63.7\% de los alumnos presentaron un IMC

- normal; al evaluar la correlación entre ambas herramientas

- con la rho de Spearman se agruparon los resultados en tres

- categorías ( 1 =subestimación, 2=normalidad y 3=sobresti-

- mación). La correlación de Spearman entre la herramienta

- de Sorensen y la de Mash y Roche fue de rho =0,695

- ( $(p=0.0)$. Conclusión: Ambas herramientas son útiles para

- evaluar la autopercepción corporal en la carrera de Diseño

- de Modas en Indumentaria y Textiles de la UAA. LUXMÉDICA,

- AÑO 11, NÚMERO 33, MAYO-AGOSTO 2016, PP 15-20.

Palabras Clave: Autopercepción corporal, distorsión, universitarios

\section{Abstract}

Autoperception is defined as the concept that an individual has about himself. It has been observed that the construction of the body image is associated with cognitive, emotional and behavioral factors. When body image does not match the body of the subject there is distortion of body image, which is related to eating disorders. Assessment tools for body perception of Sorensen and Mash and Roche present to the subject a series of male and female figures in growing progression in relation to body mass index (BMI). Objective: To evaluate the body perception tools Sorensen versus Roche Mash of college students majoring in Fashion Design in Clothing and Textiles at the Autonomous University of Aguascalientes (UAA). Materials and methods: Analytical, transverse, observational study. No random sample by quota, getting 80 students. Materials: bioimpedance scale Tanita BC-545, mechanical stadiometer SECA-2 17 format Sorensen body perception and Mash and Roche. Results: 63.7\% of students had a normal BMl; to evaluate the correlation between the two tools with Spearman rho results were grouped into three categories ( $1=$ understatement, $2=$ normal and $3=$ overestimation). Spearman correlation between the tool and Mash Sorensen and Roche was rho $=0.695(p=0.0)$. Conclusion: Both tools are useful to assess body perception in the Fashion Design and Textiles from the UAA. LUXMÉDICA, AÑO 11, NÚMERO 33, MAYO-AGOSTO 2016, PP 15-20.

Estudiantes del séptimo semestre de la Licenciatura en Nutrición del Centro de Ciencias de la Salud de la Universidad Autónoma de Aguascalientes. Maestra en Ciencias de la Nutrición, Profesora investigadora del Departamento de Nutrición y Cultura Física del Centro de Ciencias de la Salud de la Universidad Autónoma de Aguascalientes.

Fecha de recibido 29 de septiembre 2015

Fecha de aceptación: 29 de febrero 2016

Correspondencia: MCN Judith Martín del Campo Cervantes. Edificio 107, planta baja. Campus Universitario. Avenida Universidad \#940. Código postal 20131, Aguascalientes, Ags., México. Teléfono 449 9108435. Correo electrónico jmartic@correo.uaa.mx 


\section{Introducción}

La autopercepción es definida como la concepción que un individuo tiene sobre sí mismo. La imagen corporal que se forma en la mente de una persona no precisamente representa al cuerpo de forma correcta; se ha observado que su construcción está relacionada con factores cognitivos, emocionales-afectivos y conductuales ${ }^{1}$. Cuando la imagen corporal no concuerda con el cuerpo del sujeto existe una distorsión e insatisfacción de la imagen corporal, las cuales se relacionan con desórdenes alimenticios. ${ }^{2}$ Bruch en 1962 propuso por primera vez que la distorsión de la imagen corporal está evidenciada por la sobrestimación de su tamaño, siendo una característica de la anorexia nerviosa ${ }^{3}$. En México se estima que el $90 \%$ de los pacientes que padecen anorexia y bulimia son mujeres, mientras que el otro $10 \%$ son hombres; en la población universitaria, entre el 19\% y $30 \%$ de las mujeres presenta algún tipo de trastorno de la conducta alimentaria. En los últimos 20 años la prevalencia de los trastornos de la conducta alimentaria aumento en casi un $300 \%{ }^{4}$. En las herramientas de evaluación se presenta al sujeto una serie de figuras femeninas y masculinas en progresión creciente en cuanto al IMC. La escala de Sorensen presenta nueve siluetas, con una sensibilidad de 0.86 y una especificidad de $0.92^{5}$; mientras que la de Mash y Roche presenta 12 siluetas, con una sensibilidad de 0,89 y una especificidad de 0.90. ${ }^{6-10}$ Ambas tienen el objetivo de hacer una discrepancia entre el tamaño del cuerpo percibido y el tamaño del cuerpo real como medida de la distorsión. ${ }^{9}$ En la carrera de Diseño de Modas es un factor importante la imagen corporal. El objetivo de este trabajo fue realizar una comparación de las escalas de autopercepción corporal de Sorensen versus Mash y Roche.

\section{| | | | | | | | | | | | | | | | | | | | | | | | | | | | | | | | | | | | | | | | | | | | | | | | | | | | | | | | | | | | | | | | | | | | | | | | | | | | | | | | | | | | | | | | | | | | | | | | | | | | | | | | | | | | | | | | |}

\section{Material y métodos}

Estudio de tipo analítico, transversal y observacional. Muestra no probabilística por cuota conveniencia. Se incluyeron 80 alumnos de la Universidad Autónoma de Aguascalientes de la carrera de Diseño de Modas, se excluyeron a los alumnos con tratamiento psicológico y a mujeres embarazadas. Materiales: báscula de bioimpedancia Tanit BC-545 con una precisión de 100 g, estadímetro mecánico SECA-217 con una precisión de $1 \mathrm{~mm}$, formato de autopercepción corporal de Sorensen (test de Stunkard, Sorenson y Schulusinger) y de Mash y Roche. Se utilizó estadística descriptiva con el pro- grama Microsoft Office Excel 2010, y para el análisis de la estadística analítica se utilizó el Minitab 17. Primero se le permitió al alumno elegir el número de la silueta que a su consideración es más semejante a su apariencia corporal, posteriormente se pesaba y al final se medía la estatura. Para sistematizar todo, se tenía un encuestador en los test de siluetas, otra persona pesaba y otra medía (se estandarizó para poder realizar cada una de estas funciones). Las mediciones se hicieron antes de que los alumnos ingirieran alimentos y después de haber ido a orinar, para tener un peso más real. 


\section{Resultados}

Se estudió a una muestra de 80 alumnos, de los cuales el $90 \%$ correspondió al sexo femenino $(n=72)$ y el $10 \%$ al sexo masculino $(n=8)$. A cada participante se le pesó y midió, encontrando una media de peso poblacional de $60.7 \mathrm{~kg}$ (DE $\pm 15.3 \mathrm{~kg}$ ) siendo el mínimo de 39.8 kg y el máximo de 119.8 $\mathrm{kg}$; la talla poblacional tiene una media de
$163 \mathrm{~cm}(\mathrm{DE} \pm 0.07 \mathrm{~cm})$ siendo el mínimo de $147 \mathrm{~cm}$ y el máximo de $189 \mathrm{~cm}$. Al evaluar las categorías del Índice de masa corporal (IMC), se puede apreciar que de los 80 alumnos evaluados el $13.7 \%$ presenta desnutrición, el $63.7 \%$ están en el rango normal, el $11.2 \%$ tiene sobrepeso, el $7.5 \%$ se encuentra con obesidad tipo I y el 3,7\% presenta obesidad tipo II. (Figura 1)

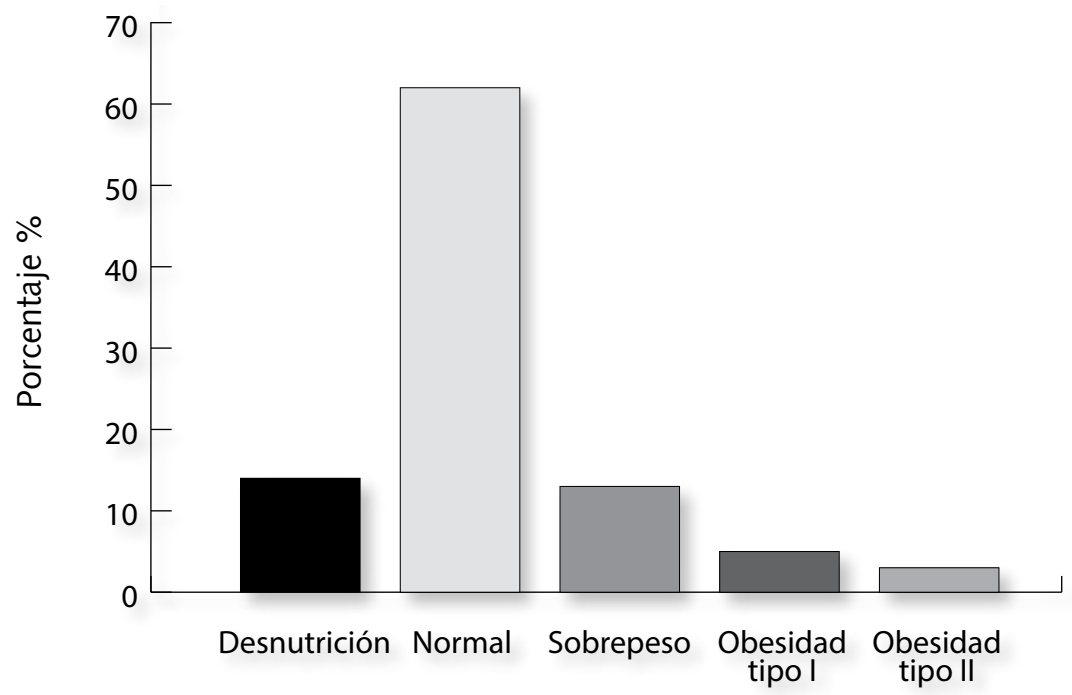

Figura 1. Distribución de las condiciones de acuerdo al resultado del índice de masa corporal de los estudiantes evaluados. $(n=80)$

La autopercepción utilizando la escala de Sorensen se muestra en la tabla 1, se observa que la silueta dos fue elegida con mayor frecuencia $(23.7 \%)$, se muestra también la proporción del IMC real que correspondió a cada silueta. (tabla 1)

\section{Tabla I}

Distribución de la autopercepción utilizando la escala de Sorensen y el porcentaje del índice de masa corporal (IMC) de los estudiantes evaluados. $\mathbf{n}=\mathbf{8 0}$

\begin{tabular}{|llrc|}
\hline & IMC (kg/m2) & $\begin{array}{c}\text { Porcentaje de la autopercepción } \\
\text { (\%) }\end{array}$ & $\begin{array}{c}\text { Porcentaje del IMC } \\
\text { (\%) }\end{array}$ \\
\hline Silueta 1 & $16-16.8$ & 12.5 & 3.7 \\
\hline Silueta 2 & $16.9-17.6$ & 23.7 & 2.5 \\
\hline Silueta 3 & $17.7-18.4$ & 18.7 & 8.7 \\
\hline Silueta 4 & $18.5-24.4$ & 5 & 62.5 \\
\hline Silueta 5 & $25-29.9$ & 11.2 & 11.2 \\
\hline Silueta 6 & $30-32.5$ & 8.7 & 5 \\
\hline Silueta 7 & $32.6-35$ & 3.7 & 3.7 \\
\hline Silueta 8 & $35.1-37.5$ & 12.5 & 2.5 \\
\hline Silueta 9 & $37.6-40$ & 3.7 & 0 \\
\hline
\end{tabular}


La autopercepción utilizando la escala de Mash y Roshe mostró los siguientes resultados: las figuras que con mayor frecuencia eligieron los participantes fueron la dos y la cuatro $(16.2 \%$ cada una de ellas) y las figuras once y doce registraron la menor frecuencia (1.2\%). Los índices de masa corporal más frecuentes fueron los que corresponden a la figuras cuatro, cinco y seis $(26.2 \%, 20 \%$ y $16.2 \%$, respectivamente). Sumando estos resultados (que abarca el rango de IMC de 18.5 hasta $24.9 \mathrm{~kg} / \mathrm{m} 2$ ) obtenemos un $62.4 \%$ que es similar al encontrado como más frecuente con la escala de Sorensen (que corresponde a la figura 4 con un rango de IMC de 18.5 a $24.4 \mathrm{~kg} / \mathrm{m} 2$ ).

\section{Tabla I}

Distribución de la autopercepción utilizando la escala de Mash y Roshe y el porcentaje del índice de masa corporal (IMC) de los estudiantes evaluados. $\mathbf{n}=80$

\begin{tabular}{|llcc|}
\hline & IMC $(\mathbf{k g} / \mathbf{m} \mathbf{)})$ & Porcentaje de la autopercepción (\%) & Porcentaje del IMC (\%) \\
\hline Silueta 1 & 16 & 12.5 & 1.2 \\
\hline Silueta 2 & $16.1-17$ & 16.2 & 3.7 \\
\hline Silueta 3 & $17.1-18.4$ & 10 & 10 \\
\hline Silueta 4 & $18.5-20.5$ & 16.2 & 26.2 \\
\hline Silueta 5 & $20.6-22.6$ & 5 & 20 \\
\hline Silueta 6 & $22.7-24.9$ & 11.2 & 16.2 \\
\hline Silueta 7 & $25-26.6$ & 8.7 & 2.5 \\
\hline Silueta 8 & $26.7-28.3$ & 3.7 & 7.5 \\
\hline Silueta 9 & $28.4-29.9$ & $5 \%$ & 1.2 \\
\hline Silueta 10 & $30-34.9$ & $8.7 \%$ & 8.7 \\
\hline Silueta 11 & $35-39.9$ & $1.2 \%$ & 2.5 \\
\hline Silueta 12 & $>40$ & $1.2 \%$ & 0 \\
\hline
\end{tabular}

Para evaluar la correlación entre ambas escalas con la rho de Spearman se agruparon los resultados en tres categorías ( 1 =subestimación, 2 =normalidad y $3=$ sobrestimación). La correlación de Spearman entre la escala de Sorensen y la de Mash y Roche fue de rho $=0,695(p=0,0)$, indicando una correlación significativa. La categorización para determinar si se subestimó, se sobrestimó o hubo una correcta percepción de la imagen corporal, fue mediante los rangos que cada silueta abarca según la herramienta que se estuviera utilizando, sin que el estudiante conociera previamente su IMC.

Las herramientas difieren en el número de siluetas que muestran, ya que la de Sorensen solamente cuenta con 9 y la de
Mash y Roshe un total de 12, lo cual en esta última, amplía el rango de cada categoría, por lo cual se puede interpretar como una herramienta con una mejor exactitud para la determinación de la imagen corporal de la persona.

El $25 \%$ de los estudiantes hombres presentaron una correcta percepción de su imagen corporal, mientras que el $37.5 \%$ la subestiman y el otro $37.5 \%$ la sobrestiman. En las estudiantes mujeres el $52.7 \%$ subestimó su imagen corporal, el $36.7 \%$ la sobrestimó y el $11.1 \%$ presentó una correcta percepción de su imagen corporal. El 37.5\% de los estudiantes subestimó su autopercepción corporal en ambas herramientas y el $32.5 \%$ de ellos sobrestimó su autopercepción corporal en ambas herramientas (figura 2). 


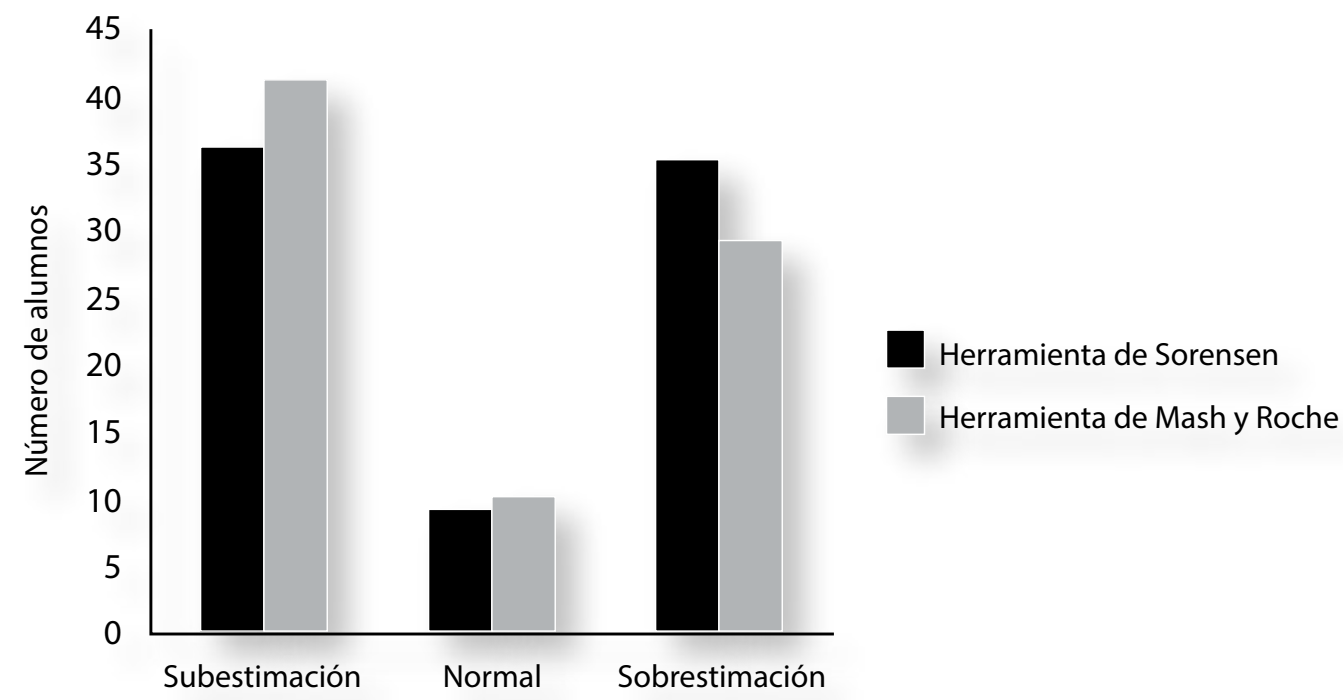

Figura 2

Comparación de la condición de subestimación, normalidad y sobre-estimación de la autopercepción obtenida con dos escalas de autopercepción diferentes. $n=80$ alumnos

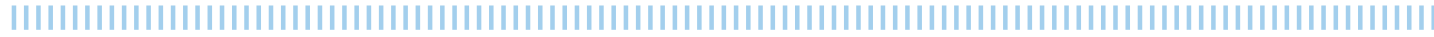

\section{Discusión}

La mayoría de los alumnos presentaron un IMC normal $(63,7 \%)$, el $13,7 \%$ presentó desnutrición, seguido por el sobrepeso con un $11,2 \%$, la obesidad tipo I con $7,5 \%$ y la obesidad tipo II con $3,7 \%$. Por lo tanto el $22,6 \%$ de la muestra presentan un IMC más alto de lo normal, quedando en el tercer lugar la desnutrición. Comparando nuestros resultados con los publicados por Saucedo Molina ${ }^{7}$ sobre conductas alimentaria de riesgo, su población mayormente presentó un IMC normal, seguido por el sobrepeso y la obesidad y en último lugar la desnutrición. Al comparar ambas poblaciones se concluye que presentan características similares, por lo que la población de estudiantes de la Licenciatura de Diseño de Modas en Indumentaria y Textiles podrían presentar un mayor riesgo para trastornos de la conducta alimentaria.

En comparación con el estudio de Osuna-Ramírez ${ }^{8}$ y la herramienta de Sorensen, en nuestra población se encontró que el $51.2 \%$ subestimó su imagen corporal y el $36.2 \%$ la sobrestimó, mientras que en ese estudio el $62.4 \%$ la sobrestimó y el $21.7 \%$ la subestimó. En nuestra población observamos que los alumnos se perciben más delgados de lo que están, siendo que el $22.6 \%$ presenta sobrepeso u obesidad, por lo que están subestimando su peso corporal. Sin embargo, el $36.2 \%$ que sobrestima su peso tiende a estar en un IMC normal o menor que éste, lo que puede favorecer la presencia de conductas relacionadas a trastornos alimentarios

En cuanto a la herramienta de Mash y Roche en el estudio Mantovanin Bruno ${ }^{6}$ se obtuvo que las mujeres en general tienden a elegir una silueta inferior a la suya, mientras que en los hombres eligen una silueta mayor que la real; en comparación con nuestra población se obtuvo que la mayoría de las mujeres $(52.7 \%$ ) eligieron una silueta inferior a la suya, y en los hombres se presentó una igualdad entre elegir una silueta superior $(37.5 \%)$ y el elegir una silueta in- 
ferior (37.5\%); esto se puede deber al número pequeño de hombres que fueron encuestados. En general se puede confirmar que las mujeres subestiman su silueta y los hombres la sobrestiman o la subestiman.

\section{Conclusiones}

Las herramientas utilizadas en este estudio para evaluar la autopercepción resultaron ser eficaces y puede utilizarse una u otra escala para poder detectar pacientes que puedan presentar conductas de riesgo alimentarias y así ser canalizados con el especialista de la salud para que se les pueda dar seguimiento.

\section{Bibliografía}

1. Vaquero-Cristóbal R, Alacid F, Muyor J, LópezMiñarro P. Imagen corporal. Nutrición Hospitalaria. 2013; 28(1):27-35.

2. Pichot $P$. Manual diagnóstico y estadístico de los trastornos mentales. In. Barcelona: Amer Psychiatric Pub; 1995. p. 553-564.

3. Gómez-Marmol A, Sánchez-Alcaraz B, MahederoNavarrete $M$. Insatisfacción y distorsión de la imagen corporal en adolescentes de doce a diecisiete años de edad. AGORA. 2013; 15:26-32.

4. Unikel-Santoncini C. Guía clínica para trastornos de la conducta alimentaria. In. México: Instituto nacional de Psiquiatria Ramón de la Fuente; 2010. p. 1417.

5. Sarabia-Cobo C. La imagen corporal en los ancianos: Estudio descriptivo. Gerokomos. 2012; 23: 15-18.
6. Mantovani B. Nivel de satisfacción con la imagen corporal en alumnos universitarios. Efdeportes. 2009; 132: 11-15

7. Saucedo-Molina T. Conductas alimentarias de riesgo, interiorización del ideal estético de delgadez e índice de masa corporal en estudiantes hidalguenses de preparatoria y licenciatura de una institución privada. Salud Mental. 2010; 33: 11-19.

8. Osuna-Ramírez I. Índice de masa corporal y percepción de la imagen corporal en una población adulta mexicana: la precisión del autoreporte. Salud Pública de México. 2006; 48: 94-103.

9. Bobadilla-Suárez S, López-Avila A. Distorsión de imagen corporal percibida e imaginada. Revista Médica del Instituto Seguro Social. 2014; 52:408-414.

10. Stunkard, AJ Sorensen, TI Schulsinger, F (eds). (1983) Use of the Danish Adoption Register for the Study of Obesity and Thinness. Raven Press: New York. 